\title{
A Novel Method to Compute the Closest Unstable Equilibrium Point for Transient Stability Region Estimate in Power Systems
}

\author{
Chih-Wen Liu and James S. Thorp, Fellow, IEEE
}

\begin{abstract}
It is well known that the threat of instability governs most aspects of modern power system operation. This being the case, any improvements made in determining the imminence of an unstable condition, or controlling it has great benefits to modern interconnected power networks. In this paper, we first review the well-known closest unstable equilibrium point (closest UEP) method for transient stability region estimate. One key step involved in the closest UEP method is to search for the closest UEP. This paper presents a new search algorithm for the closest UEP so that one can avoid the fractal nature of the Newton-Raphson method which is usually used in the closest UEP method. Finally, we applied the algorithm to three-machine system and obtained satisfactory simulation results.
\end{abstract}

Index Terms-Power systems, transient stability, UEP.

\section{INTRODUCTION}

I T IS WELL KNOWN that the threat of instability governs most aspects of modern power system operation. This being the case, any improvements made in determining the imminence of an unstable condition, or controlling it has great benefits to modern interconnected networks.

Transient stability of a power system is concerned with system's capability to withstand severe disturbance, like short circuit, lighting, and loss of generators. To determine transient stability of a power system, the conventional, and still the standard, method is to solve the system equations to obtain a time solution of the system variables and parameters, for a given scenario of events. The alternate method, which is used in this paper, is to determine stability "directly." A good survey paper on this approach is by Ribben-Pavella [1]. It appears that in order to make energy function methods practically attractive it is necessary to define "suitable" stability region estimates, i.e., estimates combining accuracy and computational efficiency. By the mid-1970's research efforts concentrated on defining such "suitable" stability region estimates $S_{k}(x)$ which would be more practical than trying to find the "theoretical" stability region $A\left(x_{s}\right)$. This resulted in the development of

Manuscript received June 29, 1995; revised September 23, 1996. This paper was recommended by Associate Editor M. Ilic.

C.-W. Liu is with the Department of Electrical Engineering, National Taiwan University, Taipei, Taiwan (e-mail: cwliu@cc.ee.ntu.edu.tw).

J. S. Thorp is with the School of Electrical Engineering, Cornell University, Ithaca, NY 14850 USA (e-mail: thorp@gonzo.ee.cornell.edu).

Publisher Item Identifier S 1057-7122(97)03484-3. the well-known closest unstable equilibrium point (closest UEP) method [1]. In [2], the stability region estimated by the closest UEP is shown to be optimal in the sense that it is the largest region within $A\left(x_{s}\right)$ which can be characterized by the corresponding energy function.

In this paper, we first review the closest UEP method. Next a novel numerical algorithm is derived and justified theoretically. Finally, we applied the algorithm to three-machine system and obtained satisfactory simulation results.

\section{MODEL, ENERgy FunCtiOnS AND THE Closest UEP Method}

In this section, we review power system dynamic models, corresponding energy functions, and the closest UEP method. We assumed that the postfault dynamic model is represented by the classical generator model with constant impedance load. We assume that the transfer admittance is purely imaginary, i.e., the system is lossless, and the transmission lines are purely reactive. For the derivation of the model, refer to [3], and for its usage in a variety of analytical studies, particularly the transient stability problem, refer to [1]-[9]. The dynamics of the $i$ th generator of a total of $n$ generators can be described by the following swing equations:

$$
\begin{aligned}
\dot{\delta}_{i} & =\omega_{i} \\
m_{i} \dot{\omega}_{i} & =P_{m i}-d_{i} \omega_{i}-\sum_{j=1}^{n} V_{i} V_{j} B_{i j} \sin \left(\delta_{i}-\delta_{j}\right)
\end{aligned}
$$

where $n$ denotes the number of generators. The parameters are as follows:

$m_{i}$ inertia constant;

$d_{i} \quad$ damping constants;

$P_{m i}$ constant mechanical power input;

$\delta_{i} \quad$ angle of internal complex voltage of $i$ th machine;

$\omega_{i} \quad$ rotor angle velocity of the $i$ th machine with respect to the reference frequency of the power system $\omega_{r}\left(\omega_{r}=\right.$ $2 \pi 60 \mathrm{rad} / \mathrm{s})$;

$B_{i j}$ transmission susceptances.

Define the function, $V(\delta, \omega)$, as the following:

$$
V(\delta, \omega)=V_{K}(\omega)+V_{P}(\delta)
$$




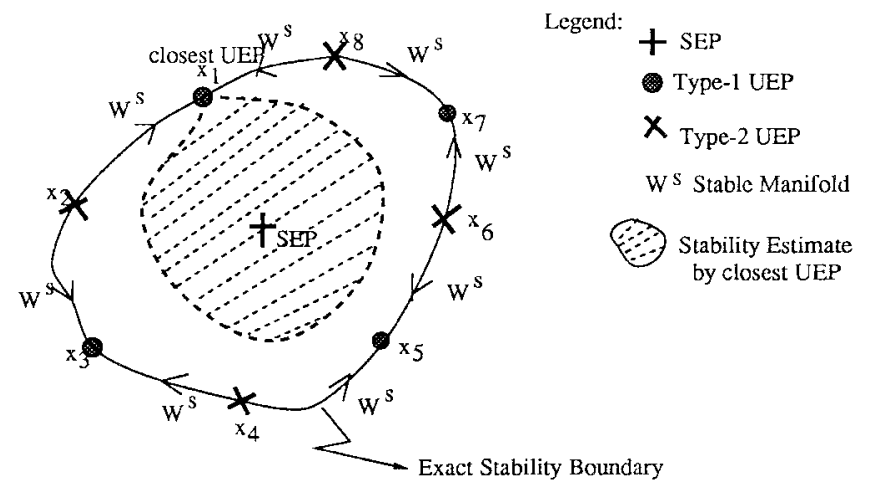

Fig. 1. The stability region estimate of the closest UEP method.

where

$$
\begin{aligned}
V_{K}(\omega) & =\frac{1}{2} \sum_{i=1}^{n} m_{i} \omega \\
V_{P}(\delta) & =-\sum_{i=1}^{n} P_{m i} \delta_{i}-\sum_{i=1}^{n} \sum_{j=1}^{n} V_{i} V_{j} B_{i j}\left[\cos \left(\delta_{i}-\delta_{j}\right)\right]
\end{aligned}
$$

where $V_{K}(\omega)$ is the kinetic energy and $V_{P}(\delta)$ is the potential energy. Differentiating the function $V(\delta, \omega)$ along the trajectories of system (2.1), (2.2) gives

$$
\dot{V}(\delta, \omega)=\frac{\partial V}{\partial \delta} \dot{\delta}+\frac{\partial V}{\partial \omega} \dot{\omega}=-\sum_{i=1}^{n} d_{i} \omega_{i}^{2} \leq 0 .
$$

This indicates that the derivative of $V(\delta, \omega)$ along the system trajectory is nonpositive. In addition, the function $V(\bullet)$ is continuously differentiable. Hence, the function $V(\delta, \omega)$ fits the definition of an energy function.

\section{A. The Closest UEP Method}

In terms of the energy functions given in the previous section, we can give the definition of the closest UEP in the state space. The definition recognized in [8] is that the closest UEP is the unstable equilibrium point having the lowest value of energy $V(\bullet)$ among all the unstable equilibrium points on the boundary of the stability region for stable equilibrium point, $x^{s}$. The following theorem shown in [8] and [9] provides an estimate of the stability region by the closest UEP method.

Theorem 2.1: Let $V(x)[:=V(\delta, \omega)]$ be the energy function. For $k \geq 0$, consider the component $S_{k}$ of the set $\{x: V(x)<k\}$ which contains the stable postfault equilibrium point $x^{s}$. Let $k_{\min }$ be the smallest $k>0$ such that $S_{k \text { min }}$ contains another equilibrium point, call it $x^{u}$. Then, $x^{u}$ is an unstable equilibrium point (UEP) and $S_{k \min } \subset A\left(x^{s}\right.$, the stability region.

The unstable equilibrium point, $x^{u}$ in (1) is the so-called closest UEP or critical UEP. In fact, the stability region estimated by the closest UEP method is optimal in the sense that this estimated region is the largest one characterized by the energy function which lies entirely within $A\left(x^{s}\right)$ [7]. A typical stability region estimate, $S_{k \text { min }}$ is illustrated in Fig. 1.

The above theorem suggests computing a stability region estimate $S_{k}$ min by the following procedure. First find all the

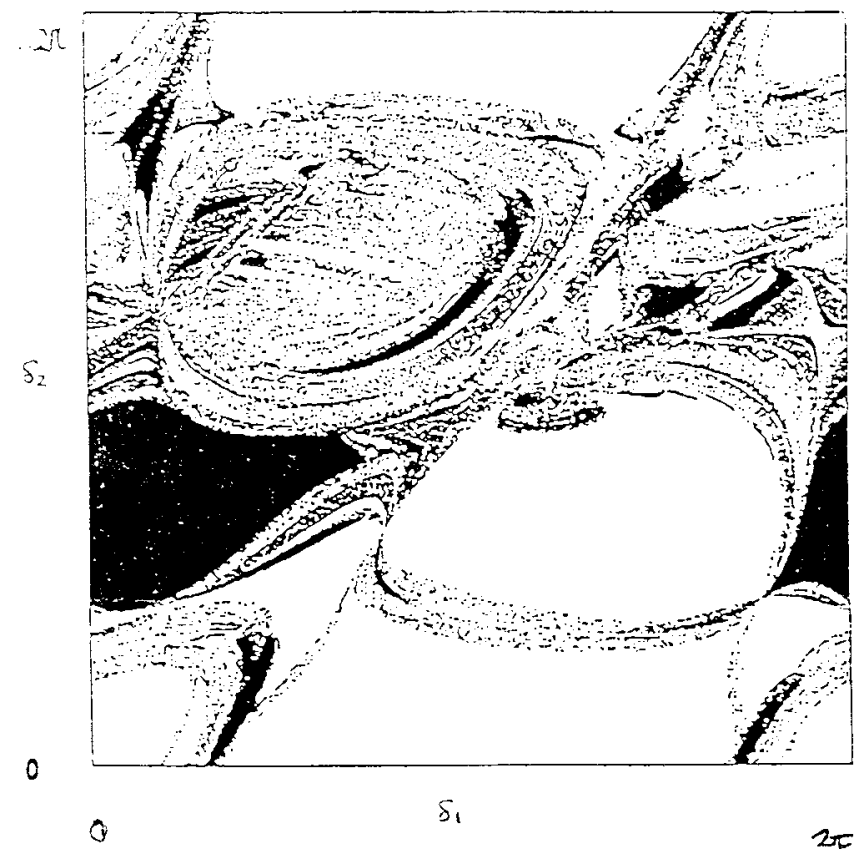

Fig. 2. Fractal nature of Newton-Raphson load flow starting points, taken from [10].

UEP's lying on the boundary $\partial A\left(\delta^{s}, \omega^{s}\right)$. Calculate $k_{\min }$ by

$$
k_{\min }=\min _{\mathrm{UEP}}\left\{V\left(\delta^{e}, \omega^{e}\right)\right\}
$$

where the minimization is over all the unstable equilibrium points (UEP) $\left(\delta^{e}, \omega^{e}\right) \in \partial A\left(\delta^{s}, \omega^{s}\right)$.

Then, $S_{k \min }\left(:=\left\{x: V(x)<k_{\min }\right\}\right)$ is a stability region estimate. The key step involved in the above procedure is finding all the UEP's on the stability boundary in order to search for the closest UEP. The next section presents an algorithm to effectively search for the closest UEP.

\section{An Algorithm for SeArching for the Closest UEP}

Finding the UEP of swing equations can be accomplished by finding the load flow solutions. Observe that $\left(\delta^{e}, \omega^{e}\right)$ is an equilibrium point of the swing equations if and only if $\omega^{e}=0$ and

$$
P_{m i}=\sum_{j=1}^{n} V_{i} V_{j} B_{i j} \sin \left(\delta_{i}^{e}-\delta_{j}^{e}\right), \quad i=1,2, \cdots, n .
$$

Equation (3.1) is just the real power flow equations, and it is difficult to determine all the solutions. It was reported that in a number cases, even if you start quite close to the UEP, and try to reach it by the Newton-Raphson iterative method, that divergence often occurs or else the solution reached is not the desired one. Thorp et al. [10] pointed out that the regions of attraction of load flow solutions calculated by Newton-Raphson have complicated boundaries which are actually fractals. The points in Fig. 2 are those initial conditions in $\delta_{1}$ and $\delta_{2}$ for a three-machine system that converge to the unstable equilibrium point (saddle) at $\delta_{1}=$ 0.4159 (radian), $\delta_{2}=1.1705$ (radian). The system has a stable equilibrium point, another saddle, and an unstable equilibrium point. 
Instead, we propose an efficient numerical method to find the closest UEP so that one can avoid the fractal nature of the Newton-Raphson method and, save computing time by not searching all the UEP's on the stability region boundary. It is observed that by using the potential energy function $V_{P}$ we can rewrite the swing equations (2.1), (2.2) as follows:

$$
\begin{aligned}
\dot{\delta}_{i} & =\omega_{i} \\
\dot{\omega}_{i} & =\frac{1}{m_{i}}\left[-d_{i} \omega_{i}-\frac{\partial V_{P}}{\partial \delta_{i}}(\delta)\right] .
\end{aligned}
$$

The above is described as the full-order swing equation. Also, $(\delta, \omega)=\left(\delta^{e}, 0\right)$ is an equilibrium of (3.2), (3.3) if and only if $\left(\partial V_{P} / \partial \delta\right)\left(\delta^{e}\right)=0$, i.e., $\delta^{e}$ is a critical point of $V_{P}$. Consider now the reduced-order gradient system

$$
\dot{\delta}=-\frac{\partial V_{P}}{\partial \delta}(\delta)
$$

Since the Jacobian matrix $J(\delta)=-\left(\partial^{2} V_{P} / \partial \delta^{2}\right)(\delta)$ is real and symmetric, it has an orthonormal set of eigenvectors $E_{1}(\delta), E_{2}(\delta), \cdots, E_{n}(\delta)$ which form a basis for $R^{n}$ space. In addition, the eigenvalues of $J(\delta)$ are real, and we can order them such that $\lambda_{1} \leq \lambda_{2} \leq \cdots \leq \lambda_{n}$. Let $P_{i}(\delta)$ be the matrix representing the orthogonal projection of a vector onto the space spanned by $E_{i}(\delta)$ :

$$
P_{i}(\delta)=E_{i}(\delta) E_{i}(\delta)^{T}
$$

Then the spectral decomposition of $J(\delta)$ is

$$
J(\delta)=\sum_{j=1}^{n} \lambda_{j}(\delta) P_{j}(\delta)
$$

and the matrices $P_{i}(\delta)$ have the following properties:

$$
\begin{aligned}
& P_{i}(\delta) P_{i}(\delta)=P_{i}(\delta) \\
& P_{i}(\delta) P_{j}(\delta)=0, \quad i \neq j .
\end{aligned}
$$

Next we create a new dynamical system as follows:

$$
\begin{aligned}
\dot{\delta}= & {\left[P_{1}(\delta)+P_{2}(\delta)+\cdots+P_{n-1}(\delta)-P n(\delta)\right] } \\
& \cdot\left[-\frac{\partial V_{P}}{\partial \delta}(\delta)\right] .
\end{aligned}
$$

The motivation in the derivation of (3.5) is that we alter the full swing equations (3.2), (3.3) to form a new system (3.5) such that all Type-1 UEP's of the full-order system become SEP's of the new system without changing the locations of equilibrium points in angle space.

Using the new dynamical system, (3.5), the proposed numerical algorithm for searching the closest UEP is outlined as follows.

Step 1.-Choosing Initial Points: Consider all $2 n$ initial points. The $j$ th and $2 j$ th initial points are expressed by

$$
\delta^{j}=\left[\delta_{1}^{s}, \delta_{2}^{s}, \cdots, \delta_{j-1}^{s}, \delta_{j}^{s}+\varepsilon, \delta_{j+1}^{s}, \cdots, \delta_{n}\right]^{T}
$$

and

$$
\delta^{2 j}=\left[\delta_{1}^{s}, \delta_{2}^{s}, \cdots, \delta_{j-1}^{s}, \delta_{j}^{s}-\varepsilon, \delta_{j+1}^{s}, \cdots, \delta_{n}\right]^{T}
$$

where $\left[\delta_{1}^{s}, \delta_{2}^{s}, \cdots, \delta_{n}^{s}\right]^{T}$ is postfault stable equilibrium point and $\varepsilon$ is a small positive perturbation angle.
Step 2.-Integrating the New Dynamical System: Integrate the new dynamical system (3.5) from initial points $\delta^{j}$ and $\delta^{2 j}$, $j=1,2, \cdots, n$. If the integration converges to a fixed point, then we store the point in a table.

Step 3.-Ordering the Points in the Table: Order the points in the table by their energy function values. The point having the lowest energy is the closest UEP.

Before using numerical examples to illustrate the effectiveness of the proposed method in the next subsection, we would like to use the following definitions and theorems to justify the method.

An equilibrium point, $\hat{x}$, is said to be type- $k$ if the number of eigenvalues of Jacobian matrix of swing equation with negative real parts is $k$. A hyperbolic equilibrium point has no purely imaginary eigenvalues of Jacobian matrix. Let $\hat{x}$ be a hyperbolic equilibrium point. Its stable and unstable manifolds are defined as follows:

$$
\begin{aligned}
W^{s}(\hat{x}) & =\left\{x \in R^{n}: \phi(x, t) \rightarrow \hat{x} \text { as } t \rightarrow \infty\right\} \\
W^{u}(\hat{x}) & =\left\{x \in R^{n}: \phi(x, t) \rightarrow \hat{x} \text { as } t \rightarrow-\infty\right\}
\end{aligned}
$$

where $\phi(x, t)$ is solution trajectory of swing equation.

The stability region (or region of attraction) of a stable equilibrium point is $x^{s}$ is defined as the following:

$$
A\left(x^{s}\right):=\left\{x \in R^{n}: \lim _{t \rightarrow \infty} \phi(x, t)=x^{s}\right\} .
$$

The boundary of stability region $A\left(x^{s}\right)$ is called the stability boundary of $x^{s}$ and will be denoted by $\partial A\left(x^{s}\right)$. Consider the set $S_{k}(x)=\left\{x \in R^{n}: V(x)<K\right\}$ where $V(\bullet): R^{n} \rightarrow R$ is continuously differentiable energy function. We call the boundary of set $S_{c}, \partial S_{c}:=\left\{x \in R^{n}: V(x)=c\right\}$ the level set and $c$ the level value.

From the general theory of differential equations we know that the stability region $A\left(x^{s}\right)$ is an open subset of the state space. Thus, from a topological viewpoint, the stability region estimate is one of estimating the boundary of stability region $\partial A\left(x^{s}\right)$. A geometrical characterization of stability boundary has been proved in [2]. It is stated as follows:

Lemma 3.1.-[2] Characterization of Stability Boundary: For a nonlinear autonomous dynamical system: $x=f[x(t)]$ which satisfies the following conditions:

i) all the equilibrium points on the stability boundary are hyperbolic;

ii) the stable and unstable manifolds of equilibrium points on stability boundary satisfy the transversality condition;

iii) every trajectory on the stability boundary approaches one of the equilibrium points as $t \rightarrow \infty$.

Let $x_{i}, i=1,2, \cdots$ be the equilibrium points on the stability boundary $\partial A\left(x^{s}\right)$ of the stable equilibrium point $x_{s}$. Then

$$
\partial A\left(x^{s}\right)=\bigcup_{i} W^{s}\left(x_{i}\right)
$$

The following two theorems based on Lemma 3.1 are critical supports for proposed algorithm. 
Theorem 3.1.-Dynamical Characterization of the Closest $U E P$ : If dynamical system satisfies the conditions of Lemma 3.1 and has an energy function $V(x)$ which is decreasing along system trajectories and the set $\{x: V[x(t)]=0\}$ does not constitute a trajectory, then the closest UEP, $x^{e}$, on the stability boundary $\partial A\left(x^{s}\right)$ is generically a Type-1 unstable equilibrium point.

Proof: We prove this theorem by contradiction. Suppose that the closest unstable equilibrium point $x^{e}$ is type- $k, k \geq 2$. Now, we consider the intersection of $W^{u}\left(x^{e}\right)$ and $\partial S_{V\left(x^{e}\right)}$. Based on Lemma 3.1, there are only two cases:

Case 1.- $\left[W^{u}\left(x^{e}\right)-x^{e}\right] \cap \partial S_{V\left(x^{e}\right)} \neq \emptyset$ : Let $p$ be a point belonging to $\left[W^{u}\left(x^{e}\right)-x^{e}\right] \cap \partial S_{V\left(x^{e}\right)}$. By the given properties of the energy function $V(x)$, we have

$$
V(p)<V\left(x^{e}\right)
$$

and also $p \in \partial S_{V\left(x^{e}\right)}$ implies

$$
V(p)=V\left(x^{e}\right) .
$$

Hence, we reach a contradiction. We preclude Case 1.

Case 2.- $\left[W^{u}\left(x^{e}\right)\right] \cap \partial S_{V\left(x^{e}\right)}=x^{e}$ : The dimension of $W^{u}\left(x^{e}\right)$ is equal to $k \geq 2$. That is, $W^{u}\left(x^{e}\right)$ is a hypersurface. Also, $\partial S_{V\left(x^{e}\right)}$ is generically a dimension $(n-1)$ hypersurface. The condition that two hypersurfaces intersect at only one point implies that $W^{u}\left(x^{e}\right)$ is tangent to $\partial S_{V\left(x^{e}\right)}$ at $x^{e}$. In a similar argument, we can get $\partial S_{V\left(x^{e}\right)}$ is tangent to $W^{s}\left(x^{e}\right)$ at $x^{e}$. So, $W^{u}\left(x^{e}\right)$ is tangent to $W^{s}\left(x^{e}\right)$ at $x^{e}$, which contradicts the assumption that $x^{e}$ is a hyperbolic equilibrium point. Therefore, the only generic value of $k$ is 1 . This completes the proof.

Theorem 3.2: If $(\delta, \omega)=\left(\delta^{e}, 0\right)$ is a type-1 UEP of fullorder system

$$
\begin{aligned}
& \dot{\delta}=\omega \\
& \dot{\omega}=M^{-1}\left[-D \omega-\frac{\partial V}{\partial \delta}(\delta)\right]
\end{aligned}
$$

then $\delta^{e}$ is a stable equilibrium point (SEP) of the new system

$$
\dot{\delta}=\left[P_{1}(\delta)+P_{2}(\delta)+\cdots+P_{n-1}(\delta)-P_{n}(\delta)\right]\left[-\frac{\partial P}{\partial \delta}(\delta)\right] .
$$

Proof: First, the proof that $\delta^{e}$ is an equilibrium point of reduced-order gradient system (3.4) is obvious. Then we prove that the equilibria of the reduced-order gradient system are the same type as the corresponding equilibria of the fullorder system. We apply the Inertia Theorem [11], which states that if $H$ is a nonsingular, symmetric matrix and $A$ has no eigenvalues on the imaginary axis, then $A H+H A^{T} \geq 0$ implies that the matrices $A$ and $H$ have the same number of eigenvalues with strictly positive and negative real parts.

The Jacobian matrix of the gradient system at $\delta^{e}$ is $-\left(\partial^{2} V_{P} / \partial \delta^{2}\right)$ and the Jacobian matrix of full system at $\left(\delta^{e}, 0\right)$ is

$$
J_{f}\left(\delta^{e}, 0\right)=\left[\begin{array}{cc}
O & I \\
-M^{-1} \frac{\partial^{2} V_{P}}{\partial \delta^{2}} & -M^{-1} D
\end{array}\right]
$$

We choose matrix $H$ to be the symmetric matrix

$$
H=\left[\begin{array}{cc}
-\left(\frac{\partial^{2} V_{P}}{\partial \delta^{2}}\right)^{-1} & O \\
O & -M^{-1}
\end{array}\right]
$$

It follows that

$$
J_{f}\left(\delta^{e}, 0\right) H+H J_{f}\left(\delta^{e}, 0\right)^{t}=\left[\begin{array}{cc}
o & o \\
o & 2 M^{-1} D M^{-1}
\end{array}\right] \geq 0 .
$$

Applying the Inertia Theorem, we see that the matrix $J\left(\delta^{e}, 0\right)$ has the same number of eigenvalues with positive real part as the matrix $-\left(\partial^{2} V_{P} / \partial \delta^{2}\right)^{-1}$ does. Since $-\left(\partial^{2} V_{P} / \partial \delta^{2}\right)^{-1}$ and $-\left(\partial^{2} V_{P} / \partial \delta^{2}\right)$ have the same number of eigenvalues with positive real parts, the matrix $J\left(\delta^{e}, 0\right)$ and the matrix $-\left(\partial^{2} V_{P} / \partial \delta^{2}\right)$ have the same number of eigenvalues with positive real part, and we prove the first part.

Finally, we prove that a type-1 UEP $\delta^{e}$ of the gradient system is a SEP of the new system. Since $\delta^{e}$ is a type-1 UEP of the gradient system, we have

$$
\begin{aligned}
0 & =-\frac{\partial V_{P}}{\partial \delta}\left(\delta^{e}\right) \\
\lambda_{1}\left(\delta^{e}\right) & <0, \cdots, \lambda_{n}\left(\delta^{e}\right)<0
\end{aligned}
$$

and

$$
\lambda_{n}\left(\delta^{e}\right)>0
$$

where $\lambda_{i}\left(\delta^{e}\right), i=1, \cdots, n$ are the eigenvalues of the Jacobian matrix, $-\left(\partial^{2} V_{P} / \partial \delta^{2}\right)$ evaluated at $\delta^{e}$. Then it is easy to show that $\delta^{e}$ is an equilibrium point of the new system

$$
\begin{aligned}
f\left(\delta^{e}\right)= & {\left[P_{1}\left(\delta^{e}\right)+P_{2}\left(\delta^{e}\right)+\cdots+P_{n-1}\left(\delta^{e}\right)-P_{n}\left(\delta^{e}\right)\right] } \\
& \cdot\left[-\frac{\partial V_{P}}{\partial \delta}\left(\delta^{e}\right)\right] \\
= & {\left[P_{1}\left(\delta^{e}\right)+P_{2}\left(\delta^{e}\right)+\cdots+P_{n-1}\left(\delta^{e}\right)-P_{n}\left(\delta^{e}\right)\right](0) } \\
= & 0 .
\end{aligned}
$$

Next we evaluate the Jacobian $J_{n}\left(\delta^{e}\right)$ of new system at $\delta^{e}$. It follows that

$$
\begin{aligned}
J_{n}\left(\delta^{e}\right)= & {\left[P_{1}\left(\delta^{e}\right)+P_{2}\left(\delta^{e}\right)+\cdots+P_{n-1}\left(\delta^{e}\right)-P_{n}\left(\delta^{e}\right)\right] } \\
& \cdot\left[-\frac{\partial^{2} V_{P}}{\partial \delta^{2}}\left(\delta^{e}\right)\right] \\
= & {\left[P_{1}\left(\delta^{e}\right)+P_{2}\left(\delta^{e}\right)+\cdots+P_{n-1}\left(\delta^{e}\right)-P_{n}\left(\delta^{e}\right)\right] } \\
& \cdot\left[\sum_{j=1}^{n} \lambda_{j}\left(\delta^{e}\right) P_{j}\left(\delta^{e}\right)\right] \\
= & {\left[\sum_{i=1}^{n-1} \lambda_{i} P_{i}\left(\delta^{e}\right)\right]-\lambda_{n} P_{n}\left(\delta^{e}\right) . }
\end{aligned}
$$

The eigenvalues of $J_{n}\left(\delta^{e}\right)$ are $\lambda_{1}, \cdots, \lambda_{n-1},\left(-\lambda_{n}\right)$ which are all negative. This implies that $\delta^{e}$ is a SEP, and completes the proof.

The principle of the proposed search algorithm is illustrated by the above theorems and the following arguments. We alter the full swing equations (3.2), (3.3) to form a new system (3.5) such that all Type-1 UEP's (including the closest UEP) of the full-order system become SEP's of the new system without changing the locations of equilibrium points in angle space. 
TABLE I

UEP's Found By the NEWTON-RAPHSON MethoD

\begin{tabular}{|c|c|c|c|}
\hline Type & $\delta_{1}$ & $\delta_{2}$ & $V_{\mathrm{P}}(\cdot)$ \\
\hline 1 & 0.0467 & 3.1149 & -0.3133 \\
\hline 1 & 0.0467 & -3.1683 & 0.3150 \\
\hline 1 & 3.0407 & 3.2232 & 1.6200 \\
\hline 2 & 2.6081 & 4.2548 & 1.7620 \\
\hline 1 & 3.2458 & 0.3341 & 1.9198 \\
\hline 2 & 3.5972 & 1.5753 & 2.0070 \\
\hline 1 & -3.0374 & 0.3341 & 2.0455 \\
\hline 2 & -2.6860 & 1.5753 & 2.1326 \\
\hline 1 & -3.2425 & -3.0600 & 2.3740 \\
\hline 2 & 2.6081 & -2.0284 & 2.4904 \\
\hline 2 & -3.6751 & -2.0284 & 2.5160 \\
\hline 2 & -2.6860 & -4.7097 & 2.7610 \\
\hline
\end{tabular}

TABLE II

Type-1 UEP's Found By the Proposed Algorithm

\begin{tabular}{|c|c|c|c|}
\hline Type & $\delta_{1}$ & $\delta_{2}$ & $\mathrm{~V}_{\mathrm{P}}(\cdot)$ \\
\hline 1 & 0.0467 & 3.1149 & -0.3133 \\
\hline 1 & 0.0467 & -3.1683 & 0.3150 \\
\hline
\end{tabular}

As to the choice of initial points, it is based on the following physical arguments. It was proposed [12], that the search for UEP's be limited to those in the proximity of the following approximate $2 n$ UEP's:

$$
\begin{gathered}
\delta^{j}=\left[\delta_{1}^{s}, \delta_{2}^{s}, \cdots, \delta_{j-1}^{s}, \delta_{j}^{s}+\pi, \delta_{j+1}^{s}, \cdots, \delta_{n}\right]^{t} \\
j=1,2, \cdots, n
\end{gathered}
$$

and

$$
\begin{gathered}
\delta^{2 j}=\left[\delta_{1}^{s}, \delta_{2}^{s}, \cdots, \delta_{j-1}^{s}, \delta_{j}^{s}-\pi, \delta_{j+1}^{s}, \cdots, \delta_{n}\right]^{T}, \\
j=1,2, \cdots, n
\end{gathered}
$$

where $\left[\delta_{1}^{s}, \delta_{2}^{s}, \cdots, \delta_{n}^{s}\right]^{T}$ is postfault stable equilibrium point. In our scheme, we replace the $\pi$ radians by the small positive angle $\varepsilon$ radians in order to guarantee that the chosen some initial points lie inside the stability region of the SEP of new system (the closest UEP of full-order system) and lie in the direction between UEP's and SEP.

\section{NUMERICAL EXAMPLES}

In this section, we illustrate the proposed numerical method for finding the closest UEP. We consider the following two examples which approximately represent a three-machine system with machine number 3 as the reference machine. Two cases with light and heavy loading are studied.

Case 1.-Light-Loading: Consider the following system:

$$
\begin{aligned}
\dot{\delta} & =\omega_{1} \\
\dot{\omega}_{1} & =-\sin \left(\delta_{1}\right)-0.5 \sin \left(\delta_{1}-\delta_{2}\right)-0.3 \omega_{1}+0.01 \\
\dot{\delta}_{2} & =\omega_{2} \\
\dot{\omega}_{2} & =-0.5 \sin \left(\delta_{2}\right)-0.5 \sin \left(\delta_{2}-\delta_{1}\right)-0.3 \omega_{1}+0.05 .
\end{aligned}
$$

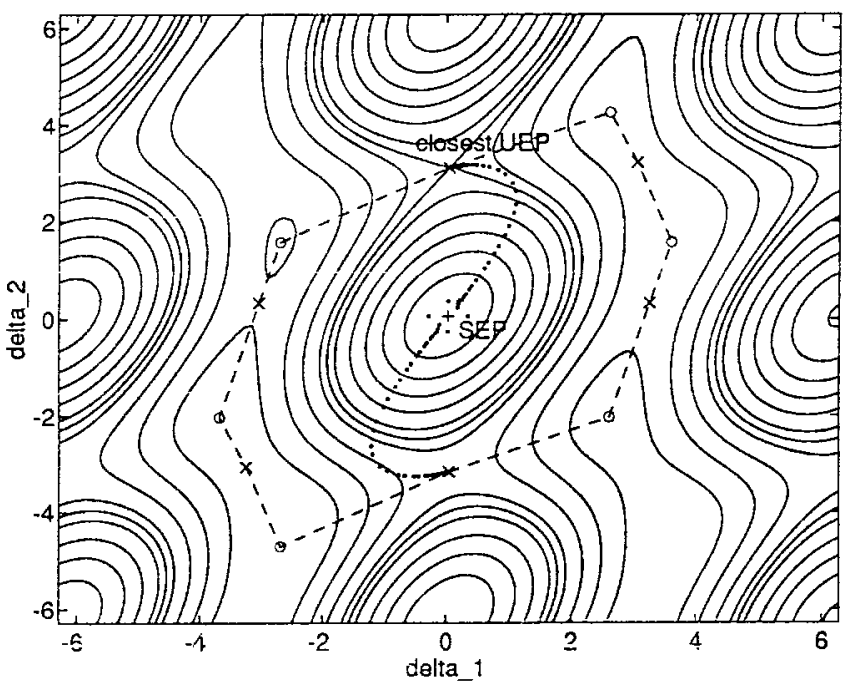

Fig. 3. The closest UEP search trajectories shown on the contour map of the potential energy. Legend: $\cdots$ search trajectory, - - exact stability boundary, + SEP, $x$ Type-1 UEP, O Type-2 UEP.

TABLE III

Type-1 UEP's Found By THE Proposed Algorithm

\begin{tabular}{|c|c|c|c|}
\hline Type & $\delta_{1}$ & $\delta_{2}$ & $\mathrm{~V}_{\mathrm{P}}(\cdot)$ \\
\hline 1 & 0.2670 & 2.9887 & -1.7334 \\
\hline 1 & 0.2670 & -3.2945 & 1.7852 \\
\hline
\end{tabular}

The corresponding reduced gradient system is

$$
\begin{aligned}
& \dot{\delta}_{1}=-\sin \left(\delta_{1}\right)-0.5 \sin \left(\delta_{1}-\delta_{2}\right)+0.01 \\
& \dot{\delta}_{2}=-0.5 \sin \left(\delta_{2}\right)-0.5 \sin \left(\delta_{2}-\delta_{1}\right)+0.05 .
\end{aligned}
$$

The energy function of system (4.2) is the following:

$$
\begin{aligned}
V\left(\delta_{1}, \delta_{2}, \omega_{1}, \omega_{2}\right)= & \omega_{1}^{2}+\omega_{2}^{2}-0.02 \delta_{1}-0.1 \delta_{2}-2 \cos \left(\delta_{1}\right) \\
& -\cos \left(\delta_{2}\right)-\cos \left(\delta_{1}-\delta_{2}\right)
\end{aligned}
$$

The point $x^{s}:=\left(\delta_{1}^{s}, \omega_{1}^{s}, \delta_{2}^{s}, \omega_{2}^{s}\right)=(0.02801,0,0.06402$, 0 ) is a SEP under study. In this system, an exhaustive search of twelve UEP's on the stability boundary by the Newton-Raphson method is given in Table I. It is observed that the closest UEP is indeed a Type-1 UEP $\left(\delta_{1}, \delta_{2}\right)=$ (0.0467, 3.1149).

Applying the algorithm developed in the previous section to search for the closest UEP, two Type-1 UEP's on the stability boundary are found (as shown in Table II). The UEP with the lowest potential energy in Table II is $(0.0467,3.1149)$ which is the same as the result from the exhaustive Newton-Raphson method. The integration trajectories, where we used 4 initial points and first-order Euler's method with stepsize 0.5, for finding the closest UEP are shown in Fig. 3.

Case 2.-Heavy-Loading: Consider the following system:

$$
\begin{aligned}
& \dot{\delta}_{1}=\omega_{1} \\
& \dot{\omega}_{1}=-\sin \left(\delta_{1}\right)-0.5 \sin \left(\delta_{1}-\delta_{2}\right)-0.3 \omega_{1}+0.06 \\
& \dot{\delta}_{2}=\omega_{2} \\
& \dot{\omega}_{2}=-0.5 \sin \left(\delta_{2}\right)-0.5 \sin \left(\delta_{2}-\delta_{1}\right)-0.3 \omega_{1}+0.28 .
\end{aligned}
$$




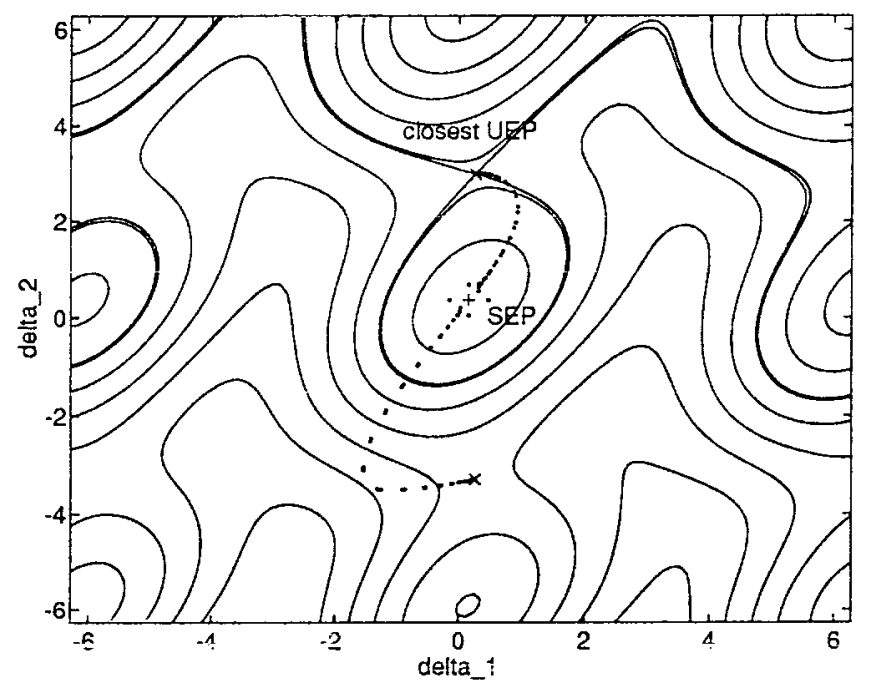

Fig. 4. The closest UEP search trajectories shown on the contour map of the potential energy. Legend: $\cdots$ search trajectory, + SEP, x Type-1 UEP.

The corresponding reduced gradient system is

$$
\begin{aligned}
& \dot{\delta}_{1}=-\sin \left(\delta_{1}\right)-0.5 \sin \left(\delta_{1}-\delta_{2}\right)+0.06 \\
& \dot{\delta}_{2}=-0.5 \sin \left(\delta_{2}\right)-0.5 \sin \left(\delta_{2}-\delta_{1}\right)+0.28
\end{aligned}
$$

The energy function of system (4.21) is the following:

$$
\begin{aligned}
& V\left(\delta_{1}, \delta_{2}, \omega_{1}, \omega_{2}\right) \\
& \quad=\omega_{1}^{2}+\omega_{2}^{2}-0.12 \delta_{1}-0.56 \delta_{2} \\
& \quad-2 \cos \left(\delta_{1}\right)-\cos \left(\delta_{2}\right)-\cos \left(\delta_{1}-\delta_{2}\right) .
\end{aligned}
$$

The point $x^{s}:=\left(\delta_{1}^{s}, \omega_{1}^{s}, \delta_{2}^{s}, \omega_{2}^{s}\right)=(0.1619,0,0.3657,0)$ is a SEP under study. In this system, only two Type-1 UEP's are on the stability boundary of the SEP, $x^{s}$. They are found by the proposed search algorithm as shown in Table III. The Type1 UEP, $\left(\delta_{1}, \delta_{2}\right)=(0.2670,2.98868)$, which has the lowest potential energy, is the closest UEP. The search trajectories are shown in Fig. 4.

\section{CONCLUding REMARKS}

Current financial and environmental trends have forced a power system to operate under more stressed conditions than in the past. The transient stability analysis is developed to provide useful information for power system control. This paper presents a new search algorithm for the closest UEP so that one can avoid the fractal nature of the Newton-Raphson method. We show that the proposed algorithm can serve as an alternative in searching for the closest UEP. Further investigation such as the utilization of the proposed technique to real power systems need to be conducted.

\section{REFERENCES}

[1] M. Ribben-Pavella and F. J. Evans, "Direct methods for studying dynamics of large scale power systems-A survey," Automatica, vol. 32, pp. 1-21, Jan. 1985.

[2] H.-D. Chiang and J. S. Thorp, "The closest unstable equilibrium point method for power system dynamic security assessment," IEEE Trans. Circuits Syst,, vol. 36, pp. 1187-1199, Dec. 1989.

[3] M. A. Pai, Energy Function Analysis for Power System Stability. Boston, MA: Kluwer, 1989.

[4] J. L. Willems and J. C. Willems, "The application of Lyapunov methods to the computation of transient stability regions for multimachine power systems," IEEE Trans. Power App. Syst., vol. PAS-89, pp. 795-801, May 1970.

[5] A. H. El-Abiad and K. Nagappan, "Transient stability region of multimachine power system," IEEE Trans. Power App. Syst., vol. PAS-85, pp. 169-178, Feb. 1966.

[6] P. C. Magnusson, "The transient energy method of calculating stability," Trans. AIEE, vol. 66, pp. 747-755, 1947.

[7] G. E. Gless, "Direct method of Lyapunov applied to transient power system stability," IEEE Trans. Power App. Syst., vol. PAS-85, pp. $159-168,1966$

[8] P. Varaiya, F. F. Wu, and R.-L. Chen, "Direct methods for transient stability analysis of power systems: Recent results," Proc. IEEE, vol. 73, pp. 1703-1715, Dec. 1985.

[9] F. M. A. Salam, "Asymptotic stability and estimating the region of attraction for the swing equations," Syst. Contr. Lett., vol. 7, no. 4, pp. 309-311, July 1986.

[10] J. S. Throp and S. A. Naqavi, "Load flow fractals," in Conf. Decision Contr., Dec. 1989, pp. 1822-1827.

[11] H. K. Wimmer, "Inertia theorems for matrices, controllability, and liner vibrations," Linear Algebra Applicat., vol. 8, pp. 337-343, 1974.

[12] M. Ribben-Pavella and B. Lemal, "Fast determination of transient stability regions for on-line power studies," Inst. Elect. Eng. Proc., vol. 123, pp. 689-696, July 1976.

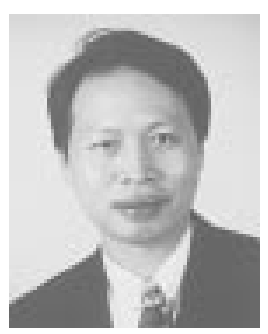

Chih-Wen Liu was born in Taiwan in 1964 . He received the B.S. degree in electrical engineering from National Taiwan University, Taipei, in 1987 and the Ph.D. degree in electrical engineering from Cornell University, Ithaca, NY, in 1994.

Since 1994, he has been with National Taiwan University, where he is an Associate Professor of electrical engineering. His research interests include power system computer applications.

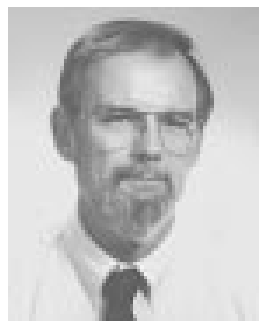

James S. Thorp (S'58-M'63-SM'80-F'89) received the B.E.E., M.S., and Ph.D. degrees from Cornell University, Ithaca, NY.

He joined the faculty of Cornell in 1962, where he is currently the Charles N. Mellowes Professor in Engineering and Director of the School of Electrical Engineering. In 1976, he was a faculty intern with the AEP Service Corporation. In 1988, he was an overseas fellow at Churchill College, Cambridge, England.

Dr. Thorp was an Associate Editor of the IEEE TRANSACTIONS ON Circuits AND Systems from 1985 to 1987 . He is a member of the National Academy of Engineering and is a member of the IEEE Power System Relaying Committee, CIGRE, Eta Kappa Nu, Tau Beta Pi, and Sigma Xi. 